\title{
P 213 USE OF A TASK AND FINISH GROUP TO IMPROVE CHILDREN'S SUPPORT BEFORE THE DEATH OF A PARENT
}

Laura Clipsham, Jane Pickard. LOROS, Leicestershire and Rutland Hospice, Leicester, UK

\subsection{6/bmjspcare-2014-000654.254}

Background In January 2013, the support of children aged 18 years and younger with a parent admitted to the hospice was audited. This showed variations in the assessment of children's needs and subsequent discussions within the multidisciplinary team, leading to the formation of a multidisciplinary Task and Finish group to review how this aspect of holistic care can be improved.

Methods A multidisciplinary group met regularly over a 6 month period. Terms of reference were agreed with the aim of reviewing identification of children's support needs, and seeking ways to improve this. Audit standards for adoption by the hospice were agreed to facilitate ongoing service evaluation. Results The group identified a need for the following:

Improved documentation: Weekly MDT meetings are a suitable forum for identifying and discussing concerns. A trial of specific prompts for record of discussions increased the documentation of children's knowledge of their parent's illness and the impact of the illness on children and their family to $100 \%$ (previously $28.6 \%$ and $71.4 \%$ respectively).

Accessible resources for patients and staff: Approval was gained from the Patient Information Group for age-appropriate leaflets to be freely accessible in the hospice, with additional child bereavement resources available if requested. Cushions showing emotions were purchased for the children's play area to improve communication with younger children.

Ongoing review of practice: A steering group was established to continue work focusing on meeting children's bereavement needs.

Conclusions Introduction of specific prompts to aid documentation of MDT discussions appear to be effective at highlighting the need for additional support for children, whilst still enabling an holistic, individualised approach to patient care. It is recommended that a specific MDT template be developed which incorporates these. The introduction of additional resources and ongoing review by a steering group will facilitate ongoing service development. 